\title{
Stimulus-response compatibility between stimulated eye and response location: implications for attentional accounts of the Simon effect
}

\author{
Fernando Valle-Inclán, Steven A. Hackley \& Carmen de Labra
}

\begin{abstract}
One influential theory of the Simon effect, the attention-shift hypothesis, states that attention movements are the origin of spatial stimulus codes. According to this hypothesis, stimulus-response compatibility effects should be absent when attention shifts are prevented. To test this prediction, we used monocular patches of color that required left or right key-press responses. About half of the subjects could discriminate which eye was stimulated (in a subsequent task), and showed strong spatial compatibility effects between the stimulated eye and the response location. The other half of the subjects could not make a utrocular discrimination (i.e., they could not judge which eye had received monocular stimulation), but the pattern of results was the same: the fastest reaction times were observed when the stimulated eye corresponded spatially to the required response (i.e., a Simon effect). Since the subjects presumably did not move their attention (from the subject's point of view, the stimuli were presented centrally), our results indicate that spatial codes can be produced in the absence of attention shifts. These results also show that utrocular discrimination can be assessed via indirect measures that are much more sensitive than explicit measures.
\end{abstract}




\section{Introduction}

Simon and Rudell (1967) presented the words 'left' and 'right' to the left or right ear and required reactions to the stimulus meaning. Although the spatial source of the sound was irrelevant, performance improved when the stimulus and response were on the same side. This phenomenon, the Simon effect, has been widely investigated. This research has yielded a general consensus in assuming that the brain produces a spatial stimulus code that, in turn, activates the spatially corresponding response (see the reviews by Lu \& Proctor, 1995, and Simon, 1990). However, how the spatial code is formed and the degree to which the activation of the compatible response should be considered an obligatory stimulus-driven process is controversial (Proctor \& Lu, 1999; Tagliabue, Zorzi, Umiltà, \& Bassignani, 2002). In this article we aimed to test the attention-shift hypothesis, a particularly influential account of how the spatial stimulus code is formed.

The attention-shift hypothesis states that the spatial stimulus code originates in the movement of the attentional spotlight (Stoffer, 1991; Umiltà \& Nicoletti, 1992). This proposition can be tested, for example, by inducing attention shifts with spatial or symbolic cues, or by preventing attention shifts by presenting task-relevant stimuli at fixation. These two approaches have generated a mixture of positive (Nicoletti \& Umiltà, 1994; Proctor \& Lu, 1994; Rubichi, Nicoletti, Iani, \& Umilità, 1997; Stoffer, 1991; Stoffer \& Yankin, 1994; Umiltà \& Liotti, 1987) and negative results (Hommel, 1993; Lamberts, Tavernier, \& D'Ydewalle, 1992; Proctor, Lu, \& van Zandt, 1992; Weeks, Chua, \& Hamblin, 1996; Zimba $\&$ Brito, 1995), and it is not clear how and why these seemingly contradictory results have arisen.

It is experimentally challenging to stabilize subjects' attention while presenting stimuli with abrupt onsets to the left or right side. A possible method is to present go-no go signals at fixation and targets at the left or right of fixation (Nicoletti \& Umiltà, 1994; Stoffer \& Yankin, 1994). However, this manipulation increases task complexity resulting in longer reaction time (RT), and it is known that the Simon effect decreases as RT increases. Thus, the reported reduction of the Simon effect using this task cannot be interpreted unambiguously as support for the attention shift hypothesis.

We attempted a novel method for preventing attention movements while providing spatial cues and keeping task complexity at the same level as in the typical Simon task. We presented monocular stimuli that were perceived as being presented at the center of the visual field, hoping that the stimulated eye could be used as a spatial cue. This strategy was based on the research on utrocular discrimination, the ability to distinguish left-eye vs. right-eye stimulation. It is known that under careful experimental conditions monocular stimuli are phenomenally identical, yet most subjects can guess above chance which eye was stimulated. This is especially true when the stimuli contain low spatial frequencies and are presented abruptly (Blake \& Cormack, 1979a, 1979b; Martens et al., 1981). Subjects typically report that they have a 'feeling' or a 'strain' in the stimulated eye and use this cue to guess which eye was stimulated. The origin of utrocular discrimination has been attributed to cues such as slight differences in the optic properties of the eyes, fixation disparities, differences in image quality or subtle differences between the physical stimuli presented to the left eye vs. right eye (e.g., Porac \& Coren, 1986). However, when these variables are controlled, monocular discrimination is still possible, which suggests that there is some type of neural code that specifies left-eye vs. right-eye stimulation (Blake \& Cormack, 1979b; Martens et al., 1981). These two interpretations of utrocular discrimination assume that there might be some signature of the eye-of-origin, even when the code is not consciously accessible and utrocular discrimination is absent. In fact, correct identification of the stimulated eye is critical for stereopsis, although normally sighted people are unaware of the monocular cues.

We wondered if the code for the eye-of-origin could be used as a spatial stimulus code. If so, monocular stimuli requiring a left or a right response might produce a Simon effect. Monocular stimuli presented within a binocularly fused space are perceived as centrally presented, and consequently the focus of attention does not move. Because of this, the Simon effect should be absent, according to the attention shift hypothesis. This outcome was reported by Craft and Simon (1970) who used a lens stereoscope and red and green light-emitting diodes as stimuli. However, the stimuli used by Craft and Simon may not have been optimal for generating a spatial code (low spatial frequencies are considered to be important, see Blake \& Cormack, 1979b; Martens et al., 1981) and they did not report if subjects were able to discriminate left-eye from right-eye stimulation. 


\section{Methods}

\section{Subjects}

Fourteen subjects with normal or corrected-to-normal vision (2 of them authors: SAH and FVI) volunteered for the experiment. All of them were right-handed and 5 were women. One subject lost fusion during the experiment and so her data were not analyzed.

\section{Procedure}

The experimental room was in complete darkness except for the light coming from the monitor (NEC Multisync, 15-inch with a refresh rate of $70 \mathrm{~Hz}$ ) placed $50 \mathrm{~cm}$ in front of the subjects. Subjects viewed the monitor through a mirror stereoscope (Geoscope Ltd, see Fig. 1). Two outline squares $(8 \times 8 \mathrm{dgv}, .1$ $\mathrm{cd} / \mathrm{m} 2$ ) were continuously presented to promote fusion. The target stimuli were red and blue gaussian patches (radius $=2 \mathrm{dgv}, .4 \mathrm{~cd} / \mathrm{m} 2$ ) presented in the center of the fusion squares. The background was black $(.004 \mathrm{~cd} / \mathrm{m} 2)$. We used gaussians to minimize possible geometrical distortions produced by the monitor and mirrors that might serve as cues for the eye-of-origin. The stimuli were presented in two pulses of 14 $\mathrm{ms}$ each (one refresh rate of the monitor), separated by $100 \mathrm{~ms}$. The reason for this pulsed presentation was to increase the chances of generating an eye-specific given that transient stimulation enhances utrocular discrimination (Martens et al., 1981). The subjects reacted by pressing one of two keys with their right index or middle finger.
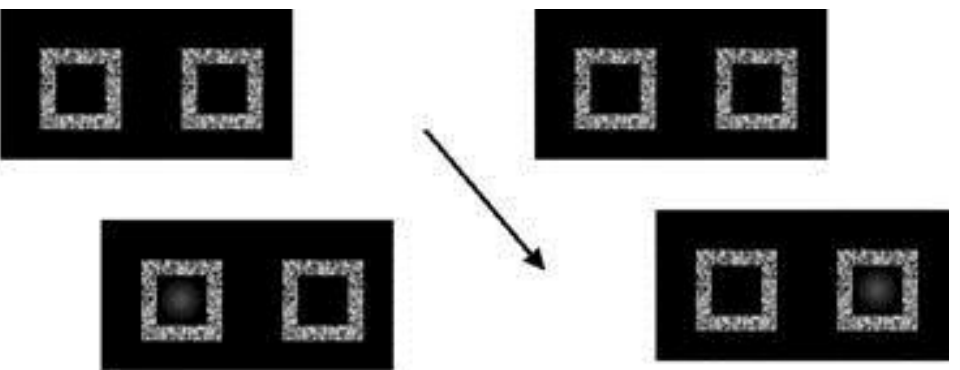

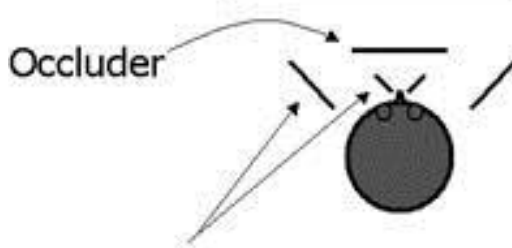

Mirrors

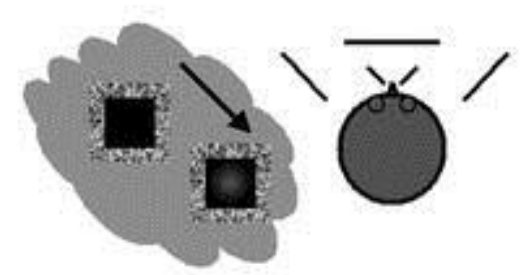

Perception

Fig.1. Schematic representation of the experimental set-up. The VGA monitor is viewed through a mirror stereoscope. Two squares are continuously presented and perceived as one square positioned in the center. Colored gaussians of $2 \mathrm{dgv}$ radius are presented to the left or right eye and subjects react according to the instructions. In the example, the color red requires a left response, so it is a compatible trial when presented to the left eye and an incompatible trial when presented to the right eye 
The experiment comprised a Simon task and an utrocular discrimination task that were identical except for the instructions. In the Simon task, subjects reacted to the color and the assignment of colors to left and right keys was counterbalanced across subjects. In the utrocular discrimination task, subjects reacted to the stimulated eye by pressing the left key for the left eye and the right key for the right eye. There was no feedback in either of the tasks. Each task consisted in six blocks of 20 trials each, with 30-s rests in between blocks. The intertrial interval varied randomly between 2,000 and 3,500 ms. All subjects performed the Simon task first.

\section{Results}

The first block of trials, and the first trial of each block were excluded from the analysis. In the utrocular discrimination task, 7 subjects correctly identified the stimulated eye well above chance ( $\geq 60 \%$ correct responses) and the other 6 were at chance levels ( $<60 \%$ correct responses, see Table 1$)$.

For the Simon task, trials with reactions faster than $100 \mathrm{~ms}$ or slower than $800 \mathrm{~ms}$ or with incorrect responses were excluded. The subjects were classified according to their performance in the utrocular discrimination task as having the ability (percentage of hits $\geq 60 \%$ ) or lacking it (percentage of hits $\approx 50 \%$ ). The data were analyzed with repeated measures ANOVA with factors utrocular discrimination (2), stimulated eye (2), and response location (2). The results are summarized in Fig. 2 and Table 1. The response accuracy was very high and there were no differences between compatible and incompatible trials (see Table 1). The RT showed the typical interaction between stimulus location (left eye vs. right eye, in this case) and response location, $F(1,11)=20.21, \mathrm{p}<.001$, with no other significant factor or interaction (see Fig. 2).

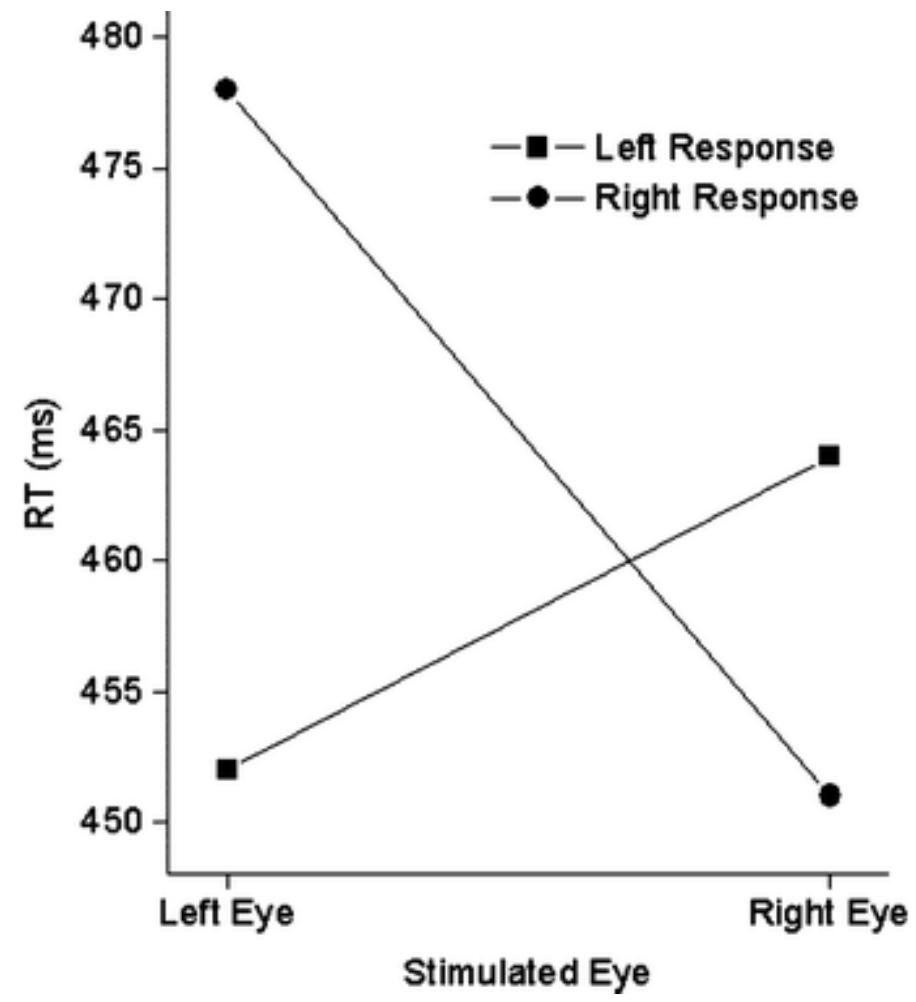

Fig. 2. Mean reaction time as a function of stimulated eye and response location 
Table 1. Percentages of correct utrocular discriminations and mean reaction time (RT) on compatible (left eye, left response; right eye, right response) and incompatible (left eye, right response; right eye, left response) trials

\begin{tabular}{lccc}
\hline Subject & $\begin{array}{l}\text { Percentage of correct } \\
\text { discriminations }\end{array}$ & $\begin{array}{l}\text { Mean RT (ms) and \% errors on } \\
\text { compatible trials }\end{array}$ & $\begin{array}{l}\text { Mean RT (ms) and \% errors on } \\
\text { incompatible trials }\end{array}$ \\
\hline & & & $471(5)$ \\
2 & .43 & $440(4)$ & $482(0)$ \\
3 & .48 & $474(4)$ & $391(4)$ \\
4 & .52 & $406(4)$ & $527(0)$ \\
5 & .52 & $504(0)$ & $525(2)$ \\
6 & .56 & $495(0)$ & $422(0)$ \\
7 & .58 & $415(4)$ & $598(7)$ \\
8 & .60 & $580(0)$ & $479(2)$ \\
9 & .60 & $461(0)$ & $464(4)$ \\
10 & .72 & $445(2)$ & $397(2)$ \\
11 & .78 & $388(2)$ & $421(4)$ \\
12 & .82 & $409(0)$ & $452(6)$ \\
13 & .82 & $420(6)$ & $508(0)$ \\
Mean & .82 & $478(6)$ & $472(2.76)$ \\
\end{tabular}

It has been reported that the Simon effect is greater when the preceding trial is compatible than when it is incompatible (e.g., Valle-Inclán, Hackley, \& de Labra, 2002). This tendency, although not significant, was also observed in the present experiment. The Simon effect was $22 \mathrm{~ms}$ when the previous trial was compatible, and $13 \mathrm{~ms}$ when the previous trial was incompatible.

\section{Discussion}

Regardless of the subjects' ability to distinguish the stimulated eye, compatible trials were faster than incompatible trials and normal sequential compatibility effects (larger Simon effect when trial N-1 was compatible than when it was incompatible) were present. These findings closely resembled those found in a typical Simon task, and suggest that the Simon effect is not critically dependent on awareness of stimulus location. Since subjects perceived the stimuli as being presented centrally and did not move their focus of attention, the results contradicted predictions based on the attention shift hypothesis. To our knowledge, this is the first time positive results have been obtained that disagree with the attention shift hypothesis. Previous contradictory evidence came from failures to replicate or were based on acceptance of the null hypothesis (see references in the Introduction section).

The attention shift hypothesis could account for the present results assuming that subjects have a cyclopean focus of attention that is moved to the left or right eye when stimuli are presented. However, this would constitute a major revision of the theory and it would involve a new attention phenomenon that has not been yet documented. However, it should be emphasized that our results do not rule out the possibility that when movements of attention do occur, as presumably they do in typical Simon tasks, they may produce spatial codes.

A second implication of these results is that spatial compatibility effects between the eye and the required response constitute a measure of utrocular discrimination that is considerably more sensitive than direct questioning. All subjects but one who did not perform above chance in the utrocular discrimination task showed Simon effect. These results strongly supported the notion of an eye-specific neural code, be it accessible to consciousness or not. It has been proposed that the basis for utrocular discrimination lies in the activity of cortical neurons biased towards one eye or the other (Blake \& Cormack, 1979b; Martens et al., 1981). However, behavioral effects of which subjects are not aware and cannot control are usually thought to be mainly subcortical. Neural responses in the lateral geniculate nucleus (LGN) or the superior colliculus are possible candidates since LGN maintains monocular segregation and the superior colliculus receives input mainly from the contralateral eye. 
This research was financed by the Spanish Ministry of Culture (PR2002-0076). We thank Briana Judd for assistance running the experiment, and Bernhad Hommel and an anonymous reviewer for their helpful suggestions for improving the paper.

\section{References}

Blake, R., \& Cormack, R. H. (1979a). Psychophysical evidence for a monocular visual cortex in stereoblind humans. Science, 203, 274-275.

Blake, R., \& Cormack, R. H. (1979b). On utrocular discrimination. Perception and Psychophysics, 26, 53-68.

Craft, J. L., \& Simon, J. R. (1970). Processing symbolic information from a visual display: interference from an irrelevant directional cue. Journal of Experimental Psychology, 83, 415-420.

Hommel, B. (1993). The role of attention for the Simon effect. Psychological Research, 55, 208-222.

Lamberts, K., Tavernier, G., \& D'Ydewalle, G. (1992). Effects of multiple reference points in spatial stimulus response compatibility. Acta Psychologica, 79, 115-130.

Lu, C. H., \& Proctor, R. W. (1995). The influence of irrelevant location information on performance: a review of the Simon and spatial Stroop effects. Psychonomic Bulletin and Review, 2, 174-207.

Martens, W., et al. (1981). What masks utrocular discrimination. Perception and Psychophysics, 30, 521-532.

Nicoletti, R., \& Umiltà, C. (1994). Attention shifts produce spatial stimulus codes. Psychological Research, $56,144-150$

Porac, C., \& Coren, S. (1986). Sighting dominance and utrocular discrimination. Perception and

Proctor, R. W., \& Lu, C.-H. (1994). Referential coding and attention-shifting accounts of the Simon

Proctor, R. W., \& Lu, C.-H. (1999). Processing irrelevant location information: practice and transfer effects in choice-reaction tasks. Memory and Cognition, 27, 63-77.

Proctor, R. W., Lu, C. H., \& Van Zandt, T. (1992). Enhancement of the Simon effect by response precuing. Acta Psychologica, 81, 53-74.

Rubichi, S., Nicoletti, R., Iani, C., \& Umiltà, C. (1997). The Simon effect occurs relative to the direction of an attention shift. Journal of Experimental Psychology: Human Perception and Performance, 23, 13531364.

Simon, J. R. (1990). The effects of an irrelevant directional cue on human information processing. In R. W. Proctor, \& T. G. Reeve (Eds.), Stimulus-response compatibility: an integrated perspective (pp. 31-88). Amsterdam: North-Holland.

Simon, J. R., \& Rudell, A. P. (1967). Auditory S-R compatibility: the effect of an irrelevant cue on information processing. Journal of Applied Psychology, 51, 300-304.

Stoffer, T. H. (1991). Attentional focussing and spatial stimulus-response compatibility. Psychological Research, 53, 127-135.

Stoffer, T. H., \& Yankin, A. R. (1994). The functional role of attention for spatial coding in the Simon effect. Psychological Research, 56, 151-162.

Tagliabue, M., Zorzi, M., Umiltà, C., \& Bassignani, F. (2000). The role of long-term-memory and short-termmemory links in the Simon effect. Journal of Experimental Psychology: Human Perception and Performance, 26, 648-670.

Umiltà, C., \& Liotti, M. (1987). Egocentric and relative spatial codes in S-R compatibility. Psychological Research, 49, 81-90.

Umiltà, C., \& Nicoletti, R. (1992). An integrated model of the Simon effect. In J. Alegria et al. (Eds.), Analytic approaches to human cognition (pp. 331-350). Amsterdam: Elsevier.

Weeks, D. J., Chua, R., \& Hamblin, K. (1996). Attention shifts and the Simon effect: a failure. Psychological Research, 58, 246-253.

Zimba, L. D., \& Brito, C. F. (1995). Attention precuing and Simon effects: a test of the attention-coding account of the Simon effect. Psychological Research, 58, 102-118. 